\title{
MEZZOGIORNO ALPINO DI GIOSUÈ CARDUCCI
}

L'omaggio è esiguo, nel segno di un gesto discreto: qualche nota su un componimento breve di Giosuè Carducci, un poeta che sembra ormai uscito dai termini un po' ondivaghi e capricciosi del canone (specie scolastico), ma che nella cornice del suo tempo ha esibito (e dentro quella cornice continua a mantenere) un suo rango, una statura robusta, forse perfino ingombrante, dalle impennate larghe e magari proterve, dal respiro sanguigno e a tutto campo. Ad ogni modo una personalità dal rilievo spiccato per il suo impegno accademico, per la sua attività di ricerca, per il ruolo pubblico assunto come poeta (e non come poeta soltanto), per la sua scrittura inesauribile, per la sua presenza salda e pugnace. Restano comunque significative le sue traduzioni dal tedesco (da Heine a von Platen), indizio di prospettive non anguste, di curiosità felici e non amidate, non scontate, sulle quali sarebbe opportuna la pausa, ma fluide e pastose sono anche le traduzioni dai classici (da Orazio, per dire), e fluida e pastosa è in genere la prosa di Carducci, armonica e composita, di grande fascino anche narrativo, oltre che per le informazioni che assicura, soprattutto l'epistolario. Il 2007, a cento anni dalla morte, ha visto un ritorno di interesse per il poeta, con manifestazioni e acquisizioni notevoli, nel dovuto rispetto della scadenza, ma è forse presto per dire se la scadenza ha inciso in profondità nella storia delle fortune critiche, se i benefici immediati del rito sono in grado di mordere negli orizzonti non sempre lineari del gusto.

Per l'occasione propongo una lettura di Mezzogiorno alpino, una poesia tarda, sulla quale ho avuto modo di ragionare in un mio corso triestino di Storia della lingua italiana e, il 1 giugno 2008, in un convegno della Canadian Society for Italian Studies a Vancouver, con remunerativi scambi di opinioni. Mezzogiorno alpino si può datare con puntualità al 27 agosto 1895, «avanti le 11», come annota lo stesso Carducci (Carducci 1935: 279), ormai sessantenne (ma lo scorcio estremo della vita di Carducci conosce un declino precipitoso), pur se il manoscritto autografo registra una variante decisiva nello stesso titolo: in prima battuta In montagna, grigio e quasi anonimo nella sua indeterminatezza, poi, fin dalla prima edizione di Rime e ritmi (Carducci 1899: 85), stadio da considerare definitivo, Mezzogiorno alpino, a incrociare le coordinate portanti dello spazio (l'arco dei monti che si imprime subito nell'aggettivo, con un sotteso perimetro di asprezze, di verdi e di silenzi) e del tempo (l'acme luminoso del giorno, l'ora topica che si raccoglie nel sostantivo). ${ }^{1}$

\footnotetext{
* Indirizzo dell'autore: Via Giulia 4, 34126 Trieste, Italia. Email: pellegri@units.it

1 Procedura analoga nel dettare le coordinate di spazio e tempo ha L'ostessa di Gaby, coeva e contigua nella raccolta: «E verde e fosca l'alpe, e limpido e fresco è il mattino...», con simmetrica disposizione degli attributi in coppia e del ricco polisindeto (Carducci 1935: 231).
} 
Mezzogiorno alpino: due quartine di endecasillabi che consentono osservazioni non approssimative sulla metrica e su una sensibilità che si apre a modi nuovi, che nel paesaggio sa trasferire una sottile capacità introspettiva, dando voce a una percezione acuta al franare ineluttabile delle cose, alla fragilità dell'esistere, alla malinconia asciutta e insieme trepida, fibrillare e stupefatta, dello scorrere e del ripiegarsi della vita. L'affiorare di risvolti umbratili e introversi, di sconcerti emotivi, di una affilata vena saturnina, che forse corregge (ma non sostituisce, non destabilizza) il ritratto del Carducci statuario, il suo anticonformismo polemico, il suo ergersi a poeta della nuova Italia, la solarità integra del vate. Non intendo mortificare qui nella sintesi banale, nella formula di comodo, una vicenda umana articolata e contraddittoria, una carriera intellettuale (e ideologica) che non rifugge dalle svolte secche, anche brusche e non indolori, ma importa ribadire una gerarchia, evitando l'equivoco: quanto emerge dai versi sotto esame resta storicamente tangenziale rispetto alla immagine rotonda, non incrinata dall'esitazione e dal dubbio, del poeta vate, senza insistere oltre sullo spettro plurimo dell'opera. Ma, dopo il preambolo protratto, ecco le due quartine di Mezzogiorno alpino:

Nel gran cerchio de l'alpi, su '1 granito

Squallido e scialbo, su' ghiacciai candenti,

Regna sereno intenso ed infinito

$\mathrm{Ne}$ '1 suo grande silenzio il mezzodí.

Pini ed abeti senza aura di venti

Si drizzano nel sol che gli penetra,

Sola garrisce in picciol suon di cetra

L'acqua che tenue tra i sassi fluí.

Accolgo qui la lezione del 1899, anno della prima stampa (con l'avvertenza: finito di stampare il 15 dicembre 1898), a dispetto (ma in qualche modo anche in forza, perché Carducci non respinge e anzi assorbe con liberalità le oscillazioni) di una leggera incongruenza: «Nel gran cerchio...» (v. 1), «nel sol che gli penetra...» (v. 6), ma «Ne 'l suo grande silenzio...» (v. 4; l'edizione nazionale interviene omologando: Carducci 1935: 230), dove la preposizione alterna forme agglutinate e forme scisse. Alla serie si affiancano «de l'alpi» (v. 1), «su 'l granito» (v. 1), mentre in «su' ghiacciai» (v. 2) l'apocope maschera (o rimuove) il fenomeno. Vischiosità della lingua letteraria, ma anche sua accusata epifania grafica, spia modestissima e pur consapevole di uno scarto: uno scarto iconico, perché la pronuncia con l'articolo in enclisi ( $N e$ 'l, su 'l) di necessità neutralizza la cesura, richiesta solo con l'articolo in proclisi («de l'alpi», dove non è consentito il ripristino della geminata). ${ }^{2}$

2 Ad allertare la mia attenzione a Vancouver su questo punto è stato Giulio C. Lepschy. Un nodo minimo, ma che può creare un qualche affanno per chi all'italiano giunga da un'altra lingua. 
Esaurisco il paragrafo delle minuzie con un cenno sulla cancellazione della vocale in uscita, ammessa (per quanto non prescritta) dopo laterale e nasale: «nel sol» (v. 6), «in picciol suon» (v. 7), ma «Si drizzano» (v. 6). Ancora nell'ordine della variazione: «Nel gran cerchio...» (v. 1), ma «Ne '1 suo grande silenzio...» (v. 4), ad assecondare armoniche diverse, ritmi più sincopati o più spediti, un dire più ruvido o decisamente maestoso. È di pertinenza morfologica il pronome gli («nel sol che gli penetra», v. 6), che a fine Ottocento si può ormai considerare desueto (Tomasin 2007: 6667; ma anche Rohlfs 1968: 162-163), mentre rientra nelle risorse contemplate dalla lingua poetica la dislocazione dell'accento: «nel sol che gli penetra» (v. 6), non sdrucciolo ma piano, in rima con cetra. Un esempio di diastole che trattiene echi precisi di memoria nobile, sciorinata nella sede di maggior prestigio, dai vv. 22-24 del ventesimo canto del Paradiso dantesco: «E come suono al collo della cetra / Prende sua forma, e sì come al pertugio / Della sampogna vento che penètra, // Così...», coppia di rime che peraltro si radica nella tradizione con filiera illustre (da Ariosto a Tasso a Parini), pur se la presenza dantesca vanta il rincalzo di altre tracce, un'altra similitudine dal secondo canto dell'Inferno, vv. 127-129, ad autorizzare il Si drizzano del v. 6 , per quanto il verbo si riferisca ad altra e più imponente essenza vegetale, alla non esile macchia dei «Pini ed abeti» (v. 5): «Quali fioretti dal notturno gelo / chinati e chiusi, poi che '1 sol li 'mbianca, / si drizzan tutti aperti in loro stelo...» (Giuliani 1999: 42). L'indugio sugli strappi più vistosi rispetto a un italiano medio, peraltro ancora virtuale, mi ha portato a sconfinare (o a immettermi) in ambiti di più specifica cogenza come la memoria letteraria.

Riprendo ora il bandolo del discorso considerando le soluzioni metriche: mai marginali per la poesia e sempre decisive in Carducci, che nel settore dimostra competenze formidabili. Un settore che conta su una bibliografia eccellente, che il taglio breve del mio contributo può solo postulare (si veda almeno Gavazzeni 1990: 207237). Ma valga preliminarmente $e$ negativo uno stralcio largo sulle odi barbare, un manifesto lucido e compiuto, in grado di illustrare le motivazioni e il ventaglio delle referenze, pur senza addentrarsi in una più spinosa tavola tecnica, nella trama comunque pungente dei sali polemici (di tangibile perspicuità l'antifrastica e certo corrucciata litania del «chiedo perdóno...», che capovolge il merito in colpa):

Queste odi poi le intitolai barbare, perché tali sonerebbero agli orecchi e al giudizio dei greci e dei romani, se bene volute comporre nelle forme metriche della loro lirica, e perché tali soneranno pur troppo a moltissimi italiani, se bene composte e armonizzate di versi e di accenti italiani. E cosi le composi, perché, avendo ad esprimere pensieri e sentimenti che mi parevano diversi da quelli che Dante, il Petrarca, il Poliziano, il Tasso, il Metastasio, il Parini, il Monti, il Foscolo, il Manzoni e il Leopardi (ricordo in specie i lirici) originalmente e splendidamente concepirono ed espressero, anche credei che questi pensieri e sentimenti io poteva esprimerli con una forma metrica meno discordante dalla forma organica con la quale mi si andavano determinando nella mente. Che se a Catullo e ad Orazio fu lecito dedurre i metri della lirica eolia nella lingua romana che altri ne aveva suoi originari, se Dante poté arricchire di care rime provenzali la poesia toscana, se di strofe francesi la arricchirono il Chiabrera e il Rinuccini, 
io dovrei secondo ragione potere sperare, che di ciò che a quei grandi poeti o a quei rimatori citati fu lode, a me si desse almeno il perdóno. Dunque chiedo perdóno dell'aver creduto che il rinnovamento classico della lirica non fosse sentenziato e finito co' tentativi per lo più impoetici di Claudio Tolomei e della sua scuola e nei pochissimi saggi del Chiabrera: chiedo perdóno del non aver disperato di questa grande lingua italiana, credendola idonea a far con essa ciò che i poeti tedeschi dal Klopstock in poi fanno assai felicemente con la loro: chiedo perdóno dell'avere osato recare qualche po' di varietà formale nella nostra lirica moderna, che non ne ha mica quel tanto che alcuni credono. Son velleità queste mie, lo so io per il primo [...]. (Carducci 1920: 530-531)

Dove importa isolare qualche passaggio: come il nesso che la metrica nuova stabilisce con un contenuto nuovo, irriducibile alle maglie codificate («avendo ad esprimere pensieri e sentimenti che mi parevano diversi... anche credei che questi pensieri e sentimenti io poteva esprimerli con una forma metrica meno discordante dalla forma organica con la quale mi si andavano determinando nella mente»), postulato ineccepibile nella sua consequenzialità, pur se metrica barbara e metrica tradizionale ammettono una qualche osmosi tematica. Ma si guardi il giudizio sommario, quasi infastidito e impaziente, sui tentativi italiani anteriori (da Tolomei a Chiabrera), accantonati per la loro inconsistenza, giudizio sommario che trova un compenso nella ribadita apertura europea, nella non scontata attenzione per 1'universo tedesco, qui riassunto nel solo (ma vigoroso) nome di Klopstock: una attenzione non genericamente indirizzata alla poesia di Klopstock, che è nota in Italia, ma al dettaglio specifico della metrica, con l'emulazione orgogliosa e fervida che ne discende. Un estro sperimentale metodico, programmato, una virtuosistica contrainte, che seleziona in relativa autonomia le sue regole, dove comunque preme segnalare (e segnalare soltanto: approfondire il capitolo della metrica barbara - e la funzione dei paradigmi tedeschi - chiederebbe altri spazi e altre conoscenze) il contatto con lo scenario europeo, ad assecondare una vitalità irrequieta e, insieme, un bisogno di applicazione scrupolosa, un rigore algido, da laboratorio, una polarità che segna - e non in superficie - l'uomo Carducci.

Mezzogiorno alpino confida nelle (o si affida alle) risorse della tradizione: due quartine di endecasillabi ineccepibili, seppur vari per ritmo, con una mobilità avvertita (sensibile la preferenza per l'ictus sulla sesta, ma l'accento risulta nitido anche sulla prima: 3, 6, $10 / 1,4,8,10 / 1,4,6,10 / 3,6,10 / / 1,4,7,10$ / 2, 6, 10 / 1, 4, 6, $8,10 / 1,4,7,10$, nell'ordine). Si direbbe un ancoraggio a un recinto di certezze, una couche riposata e appagante, una cornice di stabilità per un contenuto classicamente composto e decantato, se non marmoreo. Ma agisce già in frizione la mobilità avvertita del ritmo, il mutare della linea melodica, e ben più palesi eccentricità si colgono nello schema delle rime: ABAC BDDC, con asimmetria accusata. Il dispositivo garantisce un legame più saldo e fuso alle due quartine: queste infatti condividono non solo la rima $\mathrm{C}$, ossitona, che, come da manuale, sigilla le strofe, ma anche la $\mathrm{B}$, per quanto in sede non omologa, a produrre rima alterna nella prima quartina, baciata nella seconda. L'armonizzazione procede oltre: la rima $\mathrm{C}$ ha in comune con 
la A la vocale tonica (granito : infinito : $\operatorname{mezzod} i$ : fluí), ${ }^{3}$ che imprime un proprio timbro alla prima quartina, ma allaccia in chiusura anche la seconda, mentre la rima $\mathrm{B}$ si accorda nella vocale tonica con la D (candenti : venti : penetra : cetra), fornendo alla seconda quartina altro colore. Nei rimanti si registrerà ancora, pur nella diversità delle combinazioni, il battere della dentale (di regola sorda, sonora in mezzodí), che non investe il solo fluí, decentrato in coda. Uno scampolo di indubbia perizia, ma anche una incrinatura nel congegno perfetto, una dissonanza rattenuta. L'asimmetria è già segnale di inquietudine, di atmosfera franta e di psicologia perplessa: la tradizione, con l'archivio delle sue certezze, con la solidità dei suoi espedienti formali, non è in grado di filtrare (o di arginare) lo sconcerto emotivo, il suo trasalire, una interiorità che trema. Il sistema non regge, non assorbe la percezione di un tempo comunque precipite e non esorcizza l'ansia, a dispetto del ritegno, della ragnatela discreta e sospesa delle parole.

Mette conto insistere ancora sui rimanti, che si caricano di valore aggiunto, semantico e formale. Già si è detto della coppia penetra : cetra, dantesca, che poi si ramifica nella vicenda vertiginosa della lingua poetica. Palpabile la grana aristocratica e culta di candenti, che Carducci colloca al vertice del verso iniziale nel primo abbozzo (Ponte 1958: 67-68), ed è quindi da subito attivo nella elaborazione, in coppia con un sintagma comunque non demotico, «senza aura di venti». Anche candenti peraltro è ricco di memoria letteraria, con chiara ascendenza dantesca, dal quattordicesimo canto del Paradiso, vv. 76-78: «Oh vero sfavillar del Santo Spiro! / come si fece subito e candente / a li occhi miei che, vinti, nol soffriro!», che espone schietta la matrice latina (e in latino è impiegato da Lucrezio, Virgilio e Orazio) (Giuliani 1999: 39). Della coppia ossitona mezzodí : fluí si dirà. Nella serie si stacca granito, scabro per il referente cui si lega, non macerato dalla tradizione poetica ('esempio carducciano sfugge al censimento del Grande dizionario della lingua italiana di Salvatore Battaglia, le cui testimonianze sono di regola, se si esclude un passo di D'Annunzio, tecniche, sprovviste di alone), che qui però è abbinato a infinito, il cui profilo è inconfondibilmente marcato. Va da sé che infinito ha altre emergenze nella scrittura di Carducci (e non potrebbe essere altrimenti: la voce non ha alternative), ${ }^{4}$ ma qui il leopardismo (quasi una citazione vulgata) è accolto e smentito, declinando nel vincolo della rima (e si tratta di rima ricca: granito : infinito) le ragioni della parola vaga (e dunque della connotazione) con le istanze del termine (e dunque della denotazione), dove parola vaga e termine attingono alle precise tassonomie di Leopardi, ai contorni limpidi e perentori della sua poetica. Anche questa a suo modo una dissonanza, una massiccia e ispida immissione di vocabolario concreto, in una dialettica trasgressiva con il lessico della tradizione. Con Carducci siamo comunque lontani dal sensismo di Leopardi, dalla sua necessità di smarrimento. Ma sul rapporto complesso di Carducci con Leopardi si avrà modo di tornare.

\footnotetext{
3 Come a Vancouver mi ha fatto notare Elena Funi.

${ }^{4}$ Basti un prelievo dalle Odi barbare, Alla stazione in una mattina d'autunno: «Io voglio io voglio adagiarmi / in un tedio che duri infinito» (Carducci 1935: 97), proprio in chiusura, con cadenza che si direbbe tipica dei crepuscolari, dove anche tedio ha precedenti leopardiani.
} 
Nei versi la rete dei meccanismi iterativi è gremita, con notevole varietà (nel breve) di applicazioni: dall'anafora, che però qui disegna una figura ad anello ( $N$ Nel gran cerchio de l'alpi... Ne 'l suo grande silenzio...», vv. 1 e 4), alla allitterazione (gran... granito, v. 1, Squallido e scialbo, v. 2, «Regna sereno intenso ed infinito", v. 3, per limitare la campionatura, ma si possono aggregare almeno i ritorni della vibrante ai vv. 6 e 7, con incisiva resa acustica), alla paronomasia («nel sol... Sola garrisce...», vv. 6 e 7). Ma si osservi nella prima quartina «Nel gran cerchio... su '1 granito... su' ghiacciai... $N e$ 'l suo grande silenzio...»: un chiasmo, oscurato forse (o mimetizzato) da una aggettivazione folta, ma non ridondante, e dall'enjambement che interessa la giuntura tra primo e secondo verso, un chiasmo che isola in clausola (di frase e di strofa), a una discreta distanza dal verbo, il soggetto: «Regna... il mezzodi», con efficace e anzi lapidaria messa in rilievo. L'aggettivazione è fitta, ora anteposta al sostantivo ("gran cerchio», v. 1, "grande silenzio», v. 4), ora posposta («granito / Squallido e scialbo», "ghiacciai candenti», vv. 1-2), a confermare la figura del chiasmo. L'attributo ora è singolo («gran cerchio», v. 1, «ghiacciai candenti», v. 2, «grande silenzio», v. 4), ora aggregato in dittologia («granito / Squallido e scialbo», vv. 1-2), ma si distende anche in un ritmo ternario che alimenta il climax, un percorso in ascesa («Regna sereno intenso ed infinito», v. 3, con funzione predicativa).

L'apertura è grandiosa: «Nel gran cerchio de l'alpi...», appunto, a negare il più domestico ostacolo (e insieme abbraccio) della siepe leopardiana. Una scenografia spalancata, apparentemente oggettiva, spettacolare e pur sempre fotografica, ma al quarto verso «Ne '1 suo grande silenzio...» acquisisce già, pur nel ricorso a un aggettivo medio, immerso (e quindi reso opaco) nell'uso, ma in realtà di alta e non banale frequenza in Carducci, acquisisce già, dicevo, una risonanza interiore, il riverbero di una dimensione altra: i «sovrumani silenzi» di Leopardi. Si noti ad ogni modo il trascolorare dell'aggettivo dapprima cromatico e referenziale, pur nella gamma preziosa delle scelte, nella filigrana latina ostentata o sottesa e ammiccante: $\mathrm{i}$ «ghiacciai candenti», bianchi fino ad accecare, il «granito / Squallido e scialbo», spoglio, privo di vegetazione e quindi grigio, spento, di un bianco senza bagliori, dove l'allitterazione in evidenza è già vettore impressionistico, a incrementare il dato semantico primo. Squallido ripete il latino SQUALIDU(M), deserto, desolato, ${ }^{5}$ mentre scialbo, secondo Giovanni Ponte, contrassegna «il granito picchiettato di bianco, di

\footnotetext{
${ }^{5}$ L'aggettivo ha impiego largo in Carducci. Mi limito a un sondaggio circoscritto: «Sotto il merlato ponte scaligero, / Tra nere moli, tra squallidi alberi, / A i colli sereni, a le torri, / Onde abbrunate piangon le insegne...» (Davanti il Castel vecchio di Verona), "Il dittatore, solo, a la lugubre / Schiera davanti, ravvolto e tacito / Cavalca: la terra ed il cielo / Squallidi, plumbëi, freddi intorno» (A Giuseppe Garibaldi), «Stendonsi livide l'acque in linëa lunga che trema / Sotto squallido cielo per la lugubre macchia» (Pe'l Chiarone da Civitavecchia leggendo il Marlowe), "Ahi giorno venne quando / Morte imperando e squallido silenzio / tutto all'intorno...» (Carducci 1935: 60, 64, 101, 324).
} 
nero e di rosso» (Ponte 1958: 68). Ma non più cromatico e referenziale è l'aggettivo unito nella terna in espansione, dilatata e insieme rarefatta, "sereno intenso ed infinito", da riferire al soggetto che segue, al mezzodì (e quindi mi sembra deviante il commento di Manara Valgimigli e Giambattista Salinari: intenso «accentua l'azzurro carico e fermo della volta del cielo», mentre infinito «ne dilata lo spazio in cerchio, immagini che poi sono insieme raccolte e fuse nel grande verso, «nel suo grande silenzio il mezzodì», uno di quei divini versi che bastano da soli a creare una poesia») (Carducci 1964: 143). ${ }^{6}$

L'aggettivazione si rarefà nella seconda quartina, dove muta anche l'orientamento della sintassi, con due reggenti e due subordinate relative, a dare evidenza in punta di periodo e di componimento al cruciale fluí, un soffio e una sigla: una gnome epigrafica, per quanto implicita. L'aggettivazione si concentra nel distico finale, a staccarsi - per qualità e quantità - dai festoni sontuosi della prima quartina, in debito con pressanti ricordi latini, anche loro in qualche modo scenografici: «Sola garrisce in picciol suon di cetra / L'acqua che tenue tra i sassi fluí» (vv. 7-8), in due casi (Sola e tenue) con funzione predicativa in posizione preverbale. Sola, picciol, tenue: la prospettiva si restringe, stornando la dismisura di un orizzonte senza confini e pescando nel repertorio medio, in una ferialità disarmata (pur se per tenue Finotti annota: «la vena d'acqua è sottile, ma anche, latinamente, «chiara», «limpida», come in Ovidio, Fast., II, 250») (Segre/Ossola 1999: 457), ma imprimendo un bordone acustico, che il «grande silenzio» sottoponeva a interdizione. Del cromatismo della prima quartina non resta che una debole orma nel chiaroscuro della macchia investita dal sole: «Pini ed abeti... / nel sol che gli penetra». ${ }^{7}$

In una polarità nitida: «gran cerchio de l'alpi» e «grande silenzio» (che si propaggina nel quinto verso: «senza aura di venti») contro il «picciol suon di cetra» (e a rincalzo garrisce, tenue), con la trama leggera della allitterazione ( Sola garrisce in picciol suon di cetra...», con gli ictus ravvicinati), più stridula nella onomatopea del verbo (garrisce). Dove è indubitabile la verità del fotogramma (ottico e acustico), un fotogramma coerente e organico, che non respinge (e anzi dà corpo al) l'og-

\footnotetext{
${ }^{6}$ Mutuo dal commento di Demetrio Ferrari una suggestiva nota d'insieme di Giacinto Stiavelli, dal Fanfulla della domenica del 20 gennaio 1899. Stiavelli rileva come «ogni epiteto sia bene appropriato e giusto e come ogni frase sia una pennellata efficacissima, veramente maestra: $i l$ granito squallido e scialbo (la roccia nuda e pallida), i ghiacciai candenti (non poteva dirsi meglio per le grandi masse di ghiaccio rilucenti al sole), il mezzodì sereno, intenso, infinito (tre epiteti che dicono quanto era a dirsi) e i pini e gli abeti, la sola vegetazione del luogo, drizzantisi "nel sol che gli penetra". Dunque il sole non soltanto li illumina, né solo li colora, ma li penetra, li invade tutti, tanta è la sua intensità, tanta è la sua forza» (Carducci 1928: 179-180).

${ }^{7}$ Ma si veda il commento di Valgimigli e Salinari: «gli penètra, li avvolge, li penetra foglia per foglia, ago per ago, né foglia né ago né ramo né tronco fanno ombra da nessun lato in quella ferma luce meridiana» (Carducci 1964: 144).
} 
gettività descrittiva, la referenza piena, ma la trascende, colmandosi di un significato altro. Una polarità che trova conferma nella sintassi: non una frase unica con il soggetto in clausola e staccato dal verbo, ma un periodo più mosso e articolato, più ricco di snodi. L'attacco è nominale, nella rotondità bilanciata del ritmo binario: «Pini ed abeti...». Ma si guardi la sequenza di soggetto e predicato, includendo nello specchio le due relative che completano le reggenti: «Pini ed abeti... / $\underline{\text { Si driz- }}$ zano nel sol che gli penetra, / Sola garrisce... / L'acqua che tenue tra i sassi fluí». Per rendere più chiaro il quadro: soggetto + predicato, soggetto (pronominale) + predicato, predicato + soggetto, ma, a ostacolare la saldatura di un chiasmo complesso, che comunque nelle due principali si realizza, soggetto (pronominale) + predicato, soluzione peraltro obbligata in una relativa, dove il verbo non può precedere il pronome. Con l'effetto ultimo di dislocare nella sede di maggior prestigio l'unico passato remoto del componimento: Regna (v. 3), Si drizzano (v. 6), penetra (v. 6), garrisce (v. 7), ma fluí (v. 8).

Due sono i nuclei tematici forti e si riassumono splendidamente nei due rimanti ossitoni: il mezzodí, che rimodula il mezzogiorno del titolo, e l'acqua che fluí. Nel primo frangente non una inerte determinazione dell'ora, coordinata superficiale del tempo che si svolge, ma una fase particolare del giorno. Il sole, che è archetipo della vita, che della vita è fonte e nutrimento, è al suo acme, al culmine della sua parabola, e la natura nella sua totalità conosce una pausa, una sospensione stordita, un tempo senza tempo. È l'ora meridiana, ben messa in luce da Fabio Finotti nel suo commento (Segre/Ossola 1999: 457), l'ora panica, l'ora del sortilegio, che mette in contatto (o consente il contatto) con l'oltre, con una realtà non compresa nell'esperienza quotidiana, nell'assordante e frastornato susseguirsi dei gesti minuti, delle necessità pratiche, dei doveri imposti dalla fisiologia del corpo e dalla economia della interazione sociale. Il silenzio e l'immobilità sono presupposti irrinunciabili perché l'oltre si sveli, si renda tangibile e ammetta l'incontro momentaneo. Non fortuiti pertanto sono i sintagmi che avvolgono a cornice il mezzodí: «grande silenzio» (e solo a questo punto si percepisce lo spessore e il respiro fondo dell'attributo) e «senza aura di venti», senza alito di venti, una natura che si arresta attonita. Il motivo è appena accennato, sobrio e discreto, pur incidendosi intero nei versi, ma di lì a poco avrà modo di dispiegarsi (ma nei fatti sta già maturando) con iperbolica oltranza, nella caparbietà di una dismisura accesa, nell'Alcyone di D'Annunzio.

Protagonista (ma protagonista vereconda, nel segno di un pudore ritroso) del secondo nucleo è l'acqua, una presenza che si riduce a sommessa e smorzata colonna sonora, pur con il sostegno (unico nel componimento) di una similitudine: «Sola garrisce in picciol suon di cetra / L'acqua che tenue tra i sassi fluí». Ma anche l'acqua è archetipo della vita: l'acqua (la si intenda come liquido amniotico o come mare: il cenno è di necessità approssimativo) dà la vita. Dà la vita e quindi ha il potere di toglierla, di spegnerla, di riassorbirla, e qui si raggruma il senso del passato remoto, del fluí conclusivo. Nella elaborazione il passo è tra i più tormentati delle due quartine: 
Ma il Carducci non raggiunse sùbito un risultato così coerente: se nel v. 7 riuscì senz'altro a rendere il lieve canto del rivo ricorrendo al verbo garrire e richiamando il tintinnio della cetra, maggiori difficoltà incontrò nel successivo. Scrisse infatti dapprima L'acqua che ignota tra i massi filtrò e subito dopo aggiunse - scontento del prosastico filtrare - flù̀ uscì, superando poi l'incertezza con la definitiva scelta di flù̀, vocabolo elevato ma privo qui di ogni preziosità, e molto coerente all'esigenza di suoni liquidi e rapidi (la parola tronca accresce ora la vivacità del verso). Per soddisfare la stessa esigenza, il poeta mutò massi con sassi, e ignota (espressione qui prosastica e scialba) con tenue, parole che scorrono lievi e rapide, come fluì, e che contribuiscono quindi all'efficacia complessiva della breve lirica. (Ponte 1958: 68-69)

Dove importa soprattutto l'ingresso del passato remoto, occorrenza unica nel testo, come ho già avuto modo di sottolineare: l'acqua garrisce, in un presente che non varia, in una durata senza fratture, perché la vita comunque non si interrompe, ma poi, con scarto improvviso, con prospettiva alterata, ad archiviare una vicenda, fluí. Nel commento di Valgimigli e Salinari: «sola garrisce ecc., unico suono, quasi a dar voce al silenzio, a farcelo udire, il mormorio di un sottilissimo («tenue») rivolo di acqua che fluisce tra i sassi, anzi, che già scórse, passò, fluí» (Carducci 1964: 144). E Giuliani, nelle sue informate pagine sugli Idilli alpini: «un passato remoto di grande efficacia. Con esso il poeta, con grande senso della musicalità, vuole dirci che nel momento in cui arriva al suo orecchio il suono dell'acqua, la stessa è già andata oltre l'ostacolo, continua per la sua strada, destinata a portare il rivolo chissà dove, nel mistero della creazione» (Giuliani 1999: 45). Per questo passato remoto non mi sembra praticabile una giustificazione naturalistica. Il presente, per dire, connota il v. 13 di In Carnia (nella raccolta Rime e ritmi): «De la But che irrompe e scroscia...» (e poi ai vv. 51-62: «Sol la But tra i verdi orrori / S'ode argentëa scrosciar...»: uno degli innumerevoli specimina ritagliabili, perché la figura dell'acqua che scivola via, dolce o rapinosa, in Carducci è ricca di emergenze), in una simultaneità pacifica di filmato e colonna sonora. La traccia superstite del garrire chiederebbe ad ogni modo un passato prossimo (una azione che ha il suo avvio nel passato, ma che nel passato non si esaurisce, producendo ricadute o riverberi nel presente). ${ }^{8}$ Il passato remoto di Mezzogiorno alpino è una immissione scoperta di soggettività: la percezione del tempo umano precipite, dell'inesorabile franare dei giorni, della fugacità del vivere, della labilità estrema dell'esistere. Un sussulto di consapevolezza, pur nei termini di una composta malinconia, di una avvertita fragilità, a rimuovere le linee monumentali del personaggio pubblico, la solarità del poeta vate. Il «gran cerchio de l'alpi», il «grande silenzio» del mezzodí, l'ora panica con i suoi possibili sortilegi, cedono a uno sfondo tutto interiore e alla formula chiara della verità: un distillato di sapienza,

\footnotetext{
${ }^{8}$ A Vancouver Lepschy ha sostenuto la piena plausibilità del passato remoto (garrisce in quanto flui), ritenendola non in contrasto con la mia ipotesi interpretativa, che anzi risulterebbe confermata, ma io continuo a sentire uno strappo logico, una lacerazione sottile nello spettro delle consuetudini grammaticali.
} 
un epilogo a suo modo filosofico. In questo idillio di Carducci, ${ }^{9}$ come (ma più esplicitamente e programmaticamente) negli idilli di Leopardi. ${ }^{10}$

Il tema dell'acqua che scorre affiora anche in Sogno d'estate, che Carducci questa volta definisce idillio, nelle Odi barbare (memorabile l'incipit: «Tra le battaglie, Omero, nel carme tuo sempre sonanti / la calda ora mi vinse...»): «[...] Non più libri: la stanza da '1 sole di luglio affocata, / rintronata da i carri rotolanti su '1 ciottolato / de la città, slargossi: sorgeanmi intorno i miei colli, / cari selvaggi colli che il giovane april rifioria. / Scendeva per la piaggia con mormorii freschi un zampillo / pur divenendo rio: su '1 rio passeggiava mia madre / florida ancor ne gli anni, traendosi un pargolo a mano / cui per le spalle bianche splendevano i riccioli d'oro. / Andava il fanciulletto con piccolo passo di gloria [...]», dove l'incanto dell'infanzia (con la rassicurante e ancora giovane maglia protettiva della madre) declina con felice coerenza narrativa, con la sua distensione senza confini, la chiave dell'imperfetto, e non casualmente suppone la cornice della primavera, nello sgranarsi del polisindeto («[...] ed i pèschi ed i méli tutti eran fior' bianchi e vermigli, / e fior' gialli e turchini ridea tutta l'erba al di sotto, / ed il trifoglio rosso vestiva i declivi de' prati, / e molli d'auree ginestre si paravano i colli, / e un'aura dolce movendo quei fiori e gli odori / veniva giù da 'l mare; nel mar quattro candide vele / andavano andavano cullandosi lente nel sole [...]»). Una vita al suo primo fiorire, che si inalvea serena, in un trionfo di colori, con la tranquilla scioltezza di un fatto di natura (con parallelismo perfetto: «un zampillo / pur divenendo rio...»), senza inquietudini o perplessità nella dimensione del sogno, ma con il contrappunto del lutto nel ritorno al presente, che registra il dolore dei morti, per riscattarsi infine ancora nel respiro pieno e arioso dell'imperfetto ( Lauretta empieva intanto di gioia canora le stanze, / Bice china al telaio seguìa cheta l'opra de l'ago») (Carducci 1988: 110-111). ${ }^{11}$ Con speculare coerenza - riprendo così, dopo la digressione sullo smemorato riandare agli anni primi, il filo di Mezzogiorno alpino -, la fase del bilancio, pur pacata, ma di necessità priva di slanci, pretende altra scelta verbale.

L'epilogo è il luogo del disvelamento del significato: fluí in rapporto agonistico (un ulteriore proporsi della antitesi polare) con mezzodí, a fissare due aspetti del mondo oggettivo, ma anche, metaforicamente, l'attimo della pienezza, l'arco alto della vicen-

9 Che però Carducci non qualifica come tale: Mezzogiorno alpino non è incluso nella manciata di Idilli alpini affidati alla Nuova Antologia del 16 novembre 1898, alle pagine 193-196.

10 Carducci ha ben chiara l'individualità spiccata di Leopardi nella interpretazione del genere. Per Leopardi infatti «l'idillio non è descrizione più o meno lunga della vita agricola e pastorale nei dialoghi e nei canti, come in Teocrito in Virgilio e nel Sannazaro; e né meno rappresentazione della vita semplice di famiglia, come in Voss e nel Goethe e in altri moderni; e né meno rifacimento o ristauro di frammenti e di ricordi e di affetti antichi, come in Andrea Chenier». Leopardi «ritornò al significato e al concetto del termine greco: fece bozzetti, quadretti, ritratti istantanei d'un paesaggio, d'una impressione, d'un ricordo, d'un sogno; ma, al contrario dei greci, mettendovi dentro molto di sé e del senso e del modo onde avea percepito quella visione o subito quella impressione» (Carducci 1898: 45-46).

11 Oltre al testo allestito da Gianni A. Papini, è disponibile anche l'edizione critica commentata delle Prime odi barbare a cura di Paul van Heck, uscita anche questa nel 1988 (Leiden: Rijksuniversiteit). 
da umana, il vertice della sua parabola, e il suo esaurirsi, il suo rastremarsi, il suo declinare, il suo riflesso estremo. Negli otto versi i monosillabi sono rari: gran (v. 1), sol (v. 6), suon (v. 7), monosillabi in quanto tronchi (e da leggere nel vincolo del sintagma), e appunto fluí (che nel computo metrico però vale come bisillabo), in clauso1a. Un passato remoto che immette l'impronta decisa della soggettività, il filtro del perimetro interiore, e che con la sua momentaneità chiude a precipizio il componimento: il nesso di consonante + liquida, liscio e non vischioso, la vocale velare, con il suo timbro scuro, con il sigillo della vocale palatale, tonica, una onomatopea lacerante, una $i$ dal forte impatto acustico, con palese impressività fonosimbolica. A strozzare la curva che precede, tutta dattilica: «L'àcqua che | tènue tra i | sàssi flu | í» (in neretto le sedi toniche).

Mezzogiorno alpino: in prima battuta un foglio di diario con fresche notazioni descrittive, perfettamente vere e riconoscibili nella determinazione geografica, pur libera (e anzi sciolta) da agganci toponimici, e nella fascia del giorno. In prima battuta un idillio nella sua accezione originale di quadretto, scorcio di paesaggio, in un dettato che è pura trasparenza. Ma il testo via via si carica di (e manifesta) venature più umbratili e mosse, più inquiete e sfuggenti. Una natura come correlato oggettivo, come specchio di un io nascosto, e come premessa all'epigrafe gnomica. Un idillio nella accezione leopardiana. Carducci vate non dissolve il suo rilievo storico, la sua cifra complessiva, ma Carducci non sarebbe completo senza questa tessera (e altre simili), minima, ma irrinunciabile, che ci restituisce una personalità ripiegata e introversa, non solare, lontana dai rischi del monumento.

Resta in coda un dettaglio. Fabio Finotti, in calce alla poesia, che commenta con finezza, afferma: «L'autografo porta ancora il titolo In montagna, e la data del 27 agosto 1895: dunque posteriore a Zanella, Ora meridiana a Recoaro, che ne costituisce il più vicino modello poetico» (Segre/Ossola 1999: 457). Riporto Ora meridiana a Recoaro (Zanella 1928: 188), che è del 1872, una ode in quartine di endecasillabi e settenari, lontana dunque dal metro adottato da Carducci:

Dalla fonda vallea

D'ardui castagni giovane foresta

Al Sol drizza la testa

Che d'una pioggia d'oro li ricrea.

Una farfalla danza

Presso un rosaio: sulle lunghe sete

Della dedalea rete

Gambuto ragno ghermitor s'avanza.

Son mute l'aure; è muto

Il flauto del pastor; di quando in quando

Odo passar cantando

Fra i verdi colonnati un merlo arguto,

E fatuo re mi sembra 
Da tutti abbandonato, che passeggia

Per 1'immensa sua reggia

E d'ascoltarlo alcun non si rimembra.

I punti di contatto, che lo stesso Finotti rileva con precisione (Segre/Ossola 1999: 457), sono due: «Al Sol drizza la testa / Che d'una pioggia d'oro li ricrea» (in Carducci «Pini ed abeti senza aura di venti / Si drizzano nel sol che gli penetra», ma per Si drizzano vale ancora il precedente dantesco di cui si è detto) e «Son mute l'aure; è muto / Il flauto del pastor» (in Carducci «Regna sereno intenso ed infinito / Ne '1 suo grande silenzio il mezzodí. // Pini ed abeti senza aura di venti...»). Una contiguità che a me non sembra decisiva, comunque non in grado di mordere in profondità, di stabilire affinità e parentele non di superficie, non occasionali (e forse troppo perentorio è Finotti: «Situazione e immagini saranno riprese da Carducci in Mezzogiorno alpino»). L'ora meridiana campeggia splendidamente, con i suoi silenzi e con le sue immobilità, con le sue attonite cesure, nella leopardiana Vita solitaria, di cui riprendo uno stralcio (Leopardi 2006: 300-301). ${ }^{12}$ La situazione collima, ${ }^{13}$ a dispetto del mutato orizzonte fisico e del diverso esito che si produce, il totale obblio che astrae dalle cose e nello stesso tempo immerge nella magia del frangente («Ond'io quasi me stesso e '1 mondo obblio...»), e torna utile riferire:

Talor m'assido in solitaria parte,

Sovra un rialto, al margine d'un lago

Di taciturne piante incoronato.

Ivi quando il meriggio in ciel si volve,

La sua tranquilla imago il Sol dipinge,

Ed erba o foglia non si crolla al vento,

E non onda incresparsi, e non cicala

Strider, nè batter penna augello in ramo,

Nè farfalla ronzar, nè voce o moto

Da presso nè da lunge odi nè vedi.

Tien quelle rive altissima quiete;

Ond'io quasi me stesso e '1 mondo obblio

Sedendo immoto; e già mi par che sciolte

Giaccian le membra mie, nè spirto o senso

Piú le commova, e lor quiete antica

Coi silenzi del loco si confonda.

12 Ma si potrebbe citare anche l'Adelchi manzoniano, le stupefatte immagini del passo alpino, così remote dagli strepiti e così dense di solitudine, nel racconto del diacono Martino.

13 Sempre a Vancouver, è stato Guido Pugliese a suggerirmi l'accostamento. 
La situazione collima (analoga l'esperienza, premeranno anche le larghe fortune del motivo - dagli idilli di Teocrito a seguire in lunga teoria), ma i versi non offrono appigli incontrovertibili, dipendenze certe, prestiti, e tangenziali mi sembrano i parallelismi che si possono individuare: le essenze vegetali ( $\mathrm{Di}$ taciturne piante incoronato»), i riflessi del sole («La sua tranquilla imago il Sol dipinge»), il tacere del vento e l'atmosfera sospesa che ne discende ( «Ed erba o foglia non si crolla al vento»). La vita solitaria è indubitabilmente familiare a Carducci, ma il leopardismo di Carducci, innegabile, per quanto contrastato e rimosso, magari fortuito e involontario (e spie di questo leopardismo particolare si osservano anche in Mezzogiorno alpino: infinito che colma il ritmo ternario del terzo verso, «senza aura di venti», che si direbbe espansione di «Dolce e chiara è la notte e senza vento...», incipit di La sera del giorno festivo), in Mezzogiorno alpino non sembra contrarre debiti palesi con La vita solitaria. Una convergenza, non una propaggine: va da sé, una splendida propaggine. Ma per quelli che si definiscono idilli, sia pure alpini, e per Mezzogiorno alpino, che di questi idilli è l'espressione forse più intensa e suggestiva, il confronto con Leopardi è ineludibile: opportuno e comunque vantaggioso.

\section{Riferimenti bibliografici}

I

CARDUCCI, Giosuè (1899) Rime e ritmi. Bologna: Zanichelli.

CARDUCCI, Giosuè (1935) Odi barbare e Rime e ritmi. Bologna: Zanichelli. (Edizione nazionale delle opere, IV).

CARDUCCI, Giosuè (1928) Rime e ritmi. Con commento e note di Demetrio Ferrari. Bologna: Zanichelli.

CARDUCCI, Giosuè (1964) Rime e ritmi. Testimonianze, interpretazione, commento di Manara Valgimigli e Giambattista Salinari. Bologna: Zanichelli.

Carducci, Giosuè (1988) Odi barbare. Edizione critica a cura di Gianni A. Papini. Milano: Fondazione Arnoldo e Alberto Mondadori.

CARDUCCI, Giosuè (1898) Degli spiriti e delle forme nella poesia di Giacomo Leopardi. Bologna: Zanichelli.

CARDucCI, Giosuè (1920) Pagine di storia letteraria scelte e ordinate da Giuseppe Lipparini. Bologna: Zanichelli.

Leopardi, Giacomo (2006) Canti. Edizione critica diretta da Franco Gavazzeni. Firenze: Accademia della Crusca.

Segre, Cesare/Carlo Ossola (a cura di) (1999) Antologia della poesia italiana III: OttocentoNovecento. Torino: Einaudi.

Zanella, Giacomo (1928) Poesie. Prima edizione completa con un saggio sul poeta di Arturo Graf. Firenze: Le Monnier.

II

GavazzenI, Franco (1990) «Carducci e la metrica.» Metrica 5, 207-237.

Giuliani, Francesco (1999) L'acqua e l'alpe: gli idilli alpini del Carducci. San Severo (Foggia): Felice Miranda. 
PonTe, Giovanni (1958) «Come il Carducci compose 'Mors' 'Vignetta' 'Mezzogiorno alpino'.» La rassegna della letteratura italiana 7/1, 55-69.

RoHLFs, Gerhard (1968) Grammatica storica della lingua italiana e dei suoi dialetti. Morfologia. Torino: Einaudi.

Tomasin, Lorenzo (2007) «Classica e odierna.» Studi sulla lingua di Carducci. Firenze: Olschki.

\section{Riassunto \\ MEZZOGIORNO ALPINO DI GIOSUÈ CARDUCCI}

Il 2007, a cento anni dalla morte del poeta, ha visto un ritorno di interesse per Giosuè Carducci, personalità di grande rilievo nel secondo Ottocento per il suo impegno accademico, per la sua attività di ricerca, per il ruolo pubblico assunto come poeta, per la sua scrittura larga. Significative sono le traduzioni dal tedesco (da Heine a von Platen), ma per l'occasione si propone una rilettura di Mezzogiorno alpino, una poesia tarda: due quartine rimate che consentono rilievi non generici sulla metrica, sulle figure retoriche, sul lessico e su una sensibilità che si apre a modi nuovi, che nel paesaggio sa trasferire una sottile capacità di autoanalisi, dando voce a una percezione acuta dello scorrere del tempo e del ripiegarsi della vita.

\section{Povzetek}

MEZZOGIORNO ALPINO GIOSUÈJA CARDUCCIJA

Leta 2007, sto let po smrti Giosuèja Carduccija, se je spet pojavilo zanimanje za tega avtorja, ki je bil zaradi svojega akademskega delovanja, raziskovalnega dela, javne vloge, ki jo je prevzel kot pesnik, in zaradi izjemnega opusa ena osrednjih osebnosti druge polovice devetnajstega stoletja. Pomembni so med drugim tudi njegovi prevodi iz nemščine (od Heineja do von Platena). Avtor članka predlaga novo branje Carduccijeve pesmi iz poznega obdobja, Mezzogiorno alpino. Ta obsega dve rimani kvartini, ob katerih se porajajo številna konkretna opažanja v zvezi z metriko, rabo retoričnih figur, besediščem in občutljivostjo, ki se odpira novemu in ki skoz opazovanje pokrajine kaže pretanjeno sposobnost samoanalize, iz katere izhaja pronicljivo dojemanje minevanja časa in odtekanja življenja. 